\title{
Embedded High Precision Control and Corn Stand Counting Algorithms for an Ultra-Compact 3D Printed Field Robot
}

\author{
Erkan Kayacan*, Zhongzhong Zhang*, and Girish Chowdhary \\ Coordinated Science Laboratory \\ Distributed Autonomous Systems Laboratory \\ Department of Agricultural and Biological Engineering \\ University of Illinois at Urbana-Champaign \\ Urbana, IL 61801 USA \\ Emails: erkank@mit.edu, zzhang52@illinois.edu, girishc@illinois.edu \\ * Both authors contributed equally to this work.
}

\begin{abstract}
This paper presents embedded high precision control and corn stands counting algorithms for a low-cost, ultracompact 3D printed and autonomous field robot for agricultural operations. Currently, plant traits, such as emergence rate, biomass, vigor and stand counting are measured manually. This is highly labor intensive and prone to errors. The robot, termed TerraSentia, is designed to automate the measurement of plant traits for efficient phenotyping as an alternative to manual measurements. In this paper, we formulate a Nonlinear Moving Horizon Estimator (NMHE) that identifies key terrain parameters using onboard robot sensors and a learning-based Nonlinear Model Predictive Control (NMPC) that ensures high precision path tracking in the presence of unknown wheel-terrain interaction. Moreover, we develop a machine vision algorithm to enable TerraSentia to count corn stands by driving through the fields autonomously. We present results of an extensive field-test study that shows that (i) the robot can track paths precisely with less than $5 \mathrm{~cm}$ error so that the robot is less likely to damage plants, and (ii) the machine vision algorithm is robust against interferences from leaves and weeds, and the system has been verified in corn fields at the growth stage of V4, V6, VT, R2, and R6 from five different locations. The robot predictions agree well with the ground truth with count $_{\text {robot }}=0.96 \times$ count $_{\text {human }}+0.85$ and correlation coefficient $R=0.96$.
\end{abstract}

\section{INTRODUCTION}

Phenotypic traits are measured manually by field technicians in the field to determine physical differences between plant genotype and the influence of environmental conditions. Frequent and accurate measurement of these phenotypic traits can be utilized to breed improved crops that have more nutritional value, yield, and resilience to weather anomalies. However, manual phenotyping is expensive due to its labor-intensive nature, and prone to human measurement errors. This has led to the so-called phenotyping bottleneck preventing rapid advances in plant breeding [1], [2]. On the other hand, these crop characteristics can also be measured by exteroceptive sensors such as hyperspectral cameras, stereo cameras, thermal cameras, lidar, etc. These sensors can be integrated on a mobile robotic scanning and data processing system. Such robotic phenotyping systems have a tremendous potential to relieve the phenotyping bottleneck by significantly reducing labor cost and eliminating errors due to human-subjective information.

Unmanned robots have potential to capture extremely detailed data of plants without the need of an expert operator. A four-wheeled autonomous rover with variable chassis clearance and width is equipped with 3D time-of-flight cameras, spectral imaging, light curtain, and a laser distance sensor in [3], [4]. The rover has many promising features such as increased battery power, battery capacity, and continuous drive torque; however, its wide tracks and limited clearance are serious limitations for plant phenotyping. A new architecture consisting of two mobile robotic platforms, i.e., a phenotyping rover and a mobile observation tower, has been introduced for high-throughput field phenotyping [5]. The phenotyping rover has been built on a Husky A-200 from Clearpath Robotics and collects data from individual plants, while the observation tower identifies specific plants for further inspections. Thus, it eliminates the need of air vehicles. However, a Husky A200 is too wide (66 centimeters) and heavy (50 kilograms). A ground-based agricultural robot for high-throughput crop phenotyping was developed in [6]. It is capable of autonomously navigating below the canopy of row crops and deploying a manipulator to measure plant stalk strength and gathering phenotypic data. However, like a Husky A-200, it is too wide (56 centimeters) and heavy (140 kilograms). TerraSentia designed in this paper is much narrower (30.5 centimeters) and light (6.6 kilograms) when compared to aforementioned agricultural robots as shown in Fig. 1. Thus, it easily fits between crop-rows and does not compact the soil, which is highly desirable for improving yield.

This paper describes an ultra-compact (30 centimeters wide), ultra-light (6.6 kilograms), very low-cost and autonomous field-phenotyping robot that can navigate in a variety of field conditions to overcome the aforementioned limitations with existing field-based phenotyping systems. A wide variety of systems, e.g., tractors, unmanned aerial vehicles, rovers, and gantry systems, were developed for field-based 


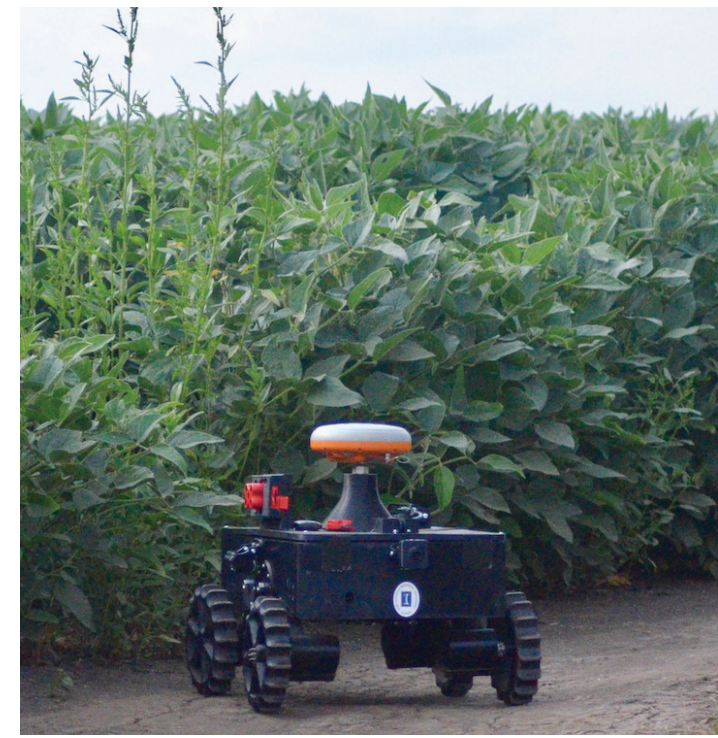

Fig. 1. The ultra-compact 3D printed field robot, termed TerraSentia, in soybean breeding plots in Energy Farm, Urbana, IL, USA.

phenotyping. However, these systems have many limitations, including high operational and maintenance costs, low coverage area, safety, logistically difficult to maintain internal combustion engines or the need for experienced operators [7]. On the other hand, the ultralight robot described here mitigates many of these challenges, yet, it leads to a uniquely challenging control problem in uneven and unstructured terrain in crop fields. The difficulties in control arise from complex and unknown wheel-terrain interaction (described in detail in Section II-A) and wheel slip. In this paper, a Nonlinear Moving Horizon Estimator (NMHE) that identifies key terrain parameters using onboard robot sensors, and a learning-based Nonlinear Model Predictive Control (NMPC) that enables high accurate tracking are developed to ensure autonomous highprecision robot mobility in off-road terrain.

The other significant contribution of this study is a novel real-time machine vision algorithm that can estimate plant stand count from image sequences obtained from a side-facing camera on an ultra-compact ground robot. This algorithm is demonstrated on the challenging problem of counting corn (Zea mays or Maize) plants in field-conditions; however, the algorithm may also be re-purposed to count other plants, including Sorghum, Wheat, Soybean, or vegetables. The algorithm leverages a cutting-edge convolution neural network architecture that runs efficiently on mobile platforms. Deep learning methods have been shown capable of recognizing complex structures and features in the presence of heavy noise. Today, deep neural networks are approaching human level at image recognition on Internet data [8]-[11]. However, no machine-vision based (whether utilizing deep-learning or not) corn stand counting algorithm currently exists that is robust to real-world noise, varying lighting conditions, and implementable in real-time on an autonomous ground robot. Moreover, the algorithm is data-efficient so that it does not need gigabytes of corn-stand data to be utilizable in practice. This is achieved through adopting transfer learning. A support vector machine (SVM) classifier is trained to classify the features extracted from a convolutional neural network with pre-trained weights. The result is a robust stand-counting algorithm where the only sensor needed is a low-cost $(<\$ 30)$ RGB camera. Our extensive field trials show that the detection is robust against noises such as corn leaves, weeds, varying lighting conditions, and residues from the previous year. Our system achieves high accuracy and reliability throughout the growing season.

This paper is organized as follows: The developed NMHENMPC framework for high precision control of the ultracompact mobile robot in off-road terrain is described in Section II. The developed real-time machine vision algorithm for corn stand counting is given in Section III. Field results are given in Section IV, and Section V concludes the paper.

\section{High Precision Control Algorithm}

\section{A. System Model}

The effect of terrain characteristics on robot performance and wheel require special attention in field-robot design because the wheels are the unique connections between the ground and robot, and nearly all forces and moments applied to the robot are transmitted through the wheels [12], [13]. Due to the wheel-terrain interaction dynamics, soil characteristic plays a vital role in determining vehicle speed and steering, which in turn are utilized for developing traction control algorithms. The knowledge of soil parameters of unknown terrain is then advantageous for improving vehicle performance [14]-[18]. In particular for lightweight robots meant for persistent field use, like TerraSentia robot described here, careful design of control algorithms is necessary to ensure that the robot does not tread over plants due to loss of precision due to unaccounted for wheel-soil interaction.

A system model including traction parameters is developed theoretically and used to establish an effective control law for the $3 \mathrm{D}$ printed field robot traveling on rough terrain in this paper. An adaptive nonlinear kinematic model is derived for the ultra-compact 3D printed field robot as an extension of the traditional kinematic model as follows:

$$
\left[\begin{array}{c}
\dot{x} \\
\dot{y} \\
\dot{\theta}
\end{array}\right]=\left[\begin{array}{c}
\mu \nu \cos \theta \\
\mu \nu \sin \theta \\
\kappa \omega
\end{array}\right]
$$

where $x$ and $y$ are the position of the field robot, $\theta$ is the yaw angle, $v$ is the wheel speed, $\omega$ is the yaw rate, and $\mu$ and $\kappa$ are the traction parameters. The difference between the traditional and developed model above is two slowly changing traction parameters. These parameters provide us a learning mechanism that can be used to minimize deviations between the real-time system and ultra-compact 3D printed field robot with an online parameter estimator. It is noted that they must be between zero and one. If the traction parameters are equal to 1 , i.e., $\mu=\kappa=1$, there exist no longitudinal or side (lateral) slips. The percentages of the longitudinal and side slips can 
be respectively found as $1-\mu$ and $1-\kappa$. It is assumed that only a fraction of the realized speed and yaw rate translates into actual vehicle motion. These fractions are given by $\mu$ and $\kappa$, and determine the effective speed $\mu \nu$ and yaw rate $\kappa \omega$. To avoid bias, these parameters should be estimated along with the full system state in each iteration based on a number of past measurements.

In this paper, the nonlinear system and measurement models are represented by the following equations:

$$
\dot{\xi}(t)=f(\xi(t), u(t), p(t)), \quad z(t)=h(\xi(t), u(t), p(t))
$$

where $\xi \in \mathbb{R}^{n_{\xi}}$ is the state vector, $u \in \mathbb{R}^{n_{u}}$ is the control input, $p \in \mathbb{R}^{n_{p}}$ is the system parameter vector, $z \in \mathbb{R}^{n_{z}}$ is the measured output, $f(\cdot, \cdot, \cdot): \mathbb{R}^{n_{\xi}+n_{u}+n_{p}} \longrightarrow \mathbb{R}^{n_{\xi}}$ is the continuously differentiable state update function and $f(0,0, p)=0 \forall t$, and $h: R^{n_{\xi}+n_{u}+n_{p}} \longrightarrow R^{n_{z}}$ is the measurement function. The derivative of $\xi$ with respect to $t$ is denoted by $\dot{\xi} \in \mathbb{R}^{n} \xi$. The state, parameter, input and output vectors are respectively denoted as follows:

$$
\begin{aligned}
& \xi=\left[\begin{array}{lll}
x & y & \theta
\end{array}\right]^{T}, \quad p=\left[\begin{array}{lll}
v & \mu & \kappa
\end{array}\right]^{T} \\
& u=\omega, \quad z=\left[\begin{array}{llll}
x & y & v & \omega
\end{array}\right]^{T}
\end{aligned}
$$

\section{B. Nonlinear Moving Horizon Estimation}

Although model-based controllers need full state and parameter information to generate a control signal, the number of sensors is less than the number of measurable states and parameters in practice. Therefore, state estimators are required to estimate immeasurable states and parameters. Extended Kalman filter is the most well-known state-estimation method for nonlinear systems. However, EKFs are not capable of dealing with constrained nonlinear systems [19]. As can be seen in (2), the traction parameter estimates play a vital role for the ultra-compact 3D printed robot, and there exist constraints on these parameters, which makes Extended Kalman filter inconvenient for our system. Nonlinear Moving Horizon Estimation (NMHE) approach has a capability of dealing with constraints on state and parameter and is formulated for the ultra-compact 3D printed robot as follows:

$$
\begin{array}{ll}
\min _{\xi(t), p, u(t)} & \frac{1}{2}\left\{\| \begin{array}{c}
\hat{\xi}\left(t_{k-N_{e}+1}\right)-\xi\left(t_{k-N_{e}+1}\right) \\
\hat{p}-p
\end{array}\right. \\
& \left.+\sum_{i=k-N_{e}+1}^{k}\left\|z_{m}\left(t_{i}\right)-z\left(t_{i}\right)\right\|_{H_{k}}^{2}\right\} \\
\text { s. t. } & \dot{\xi}(t)=f(\xi(t), u(t), p) \\
& z(t)=h(\xi(t), u(t), p) \\
& 0 \leq \mu \text { and } \kappa \leq 1 \quad \forall t \in\left[t_{k-N_{e}}, t_{k}\right]
\end{array}
$$

where the deviations of the state and parameter estimates before the estimation horizon are minimized by a symmetric positive semi-definite weighting matrix $H_{N}$, and the deviations of the measured and system outputs in the estimation horizon are minimized by a symmetric positive semi-definite weighting matrix $H_{k}$ [20]. The estimation horizon is represented by $N_{e}$, and lower and upper bounds on the traction parameters $\mu \kappa$ are respectively defined as 0 and 1 . The objective function in the NMHE formulation consists of two parts: the arrival and quadratic costs. The arrival cost stands for the early measurements $t=\left[t_{0, k-N_{e}}\right]$, and the quadratic cost stands for the recent measurements $t=\left[t_{k-N_{e}+1, k}\right]$.

The measurements have been perturbed by Gaussian noise with standard deviation of $\sigma_{x}=\sigma_{y}=0.03 \mathrm{~m}, \sigma_{\omega}=0.0175$ $\mathrm{rad} / \mathrm{s}, \sigma_{v}=0.05 \mathrm{~m} / \mathrm{s}$ based on experimental analysis. Therefore, the following weighting matrices $H_{k}$ and $H_{N}$ are used in the NMHE:

$$
\begin{aligned}
H_{k} & =\operatorname{diag}\left(\sigma_{x}^{2}, \sigma_{y}^{2}, \sigma_{v}^{2}, \sigma_{\omega}^{2}\right)^{-1} \\
& =\operatorname{diag}\left(0.03^{2}, 0.03^{2}, 0.5^{2}, 0.35^{2}\right)^{-1} \\
H_{N} & =\operatorname{diag}\left(x^{2}, y^{2}, \theta^{2}, v^{2}, \mu^{2}, \kappa^{2}\right)^{-1} \\
& =\operatorname{diag}\left(10.0^{2}, 10.0^{2}, 0.1^{2}, 1.0^{2}, 0.25^{2}, 0.25^{2}\right)^{-1}
\end{aligned}
$$

The inputs to the NMHE algorithm are the position values coming from the global navigation satellite system, the velocity values coming from the encoders mounted on the DC motors and the yaw rate values coming from the gyro. The outputs of NMHE are the position in $\mathrm{x}$ - and y-coordinate system, the yaw angle, the wheel velocity and the traction coefficients. The estimated values are then fed to the NMPC.

\section{Nonlinear Model Predictive Control}

Nonlinear Model Predictive Control (NMPC) approach is the most well known control method for system with fast dynamics. The reason is that NMPC has the capability of dealing with hard constraints on state and input, and online optimization allows updating cost, model parameters, constraints [21], [22]. The following finite horizon optimal control formulation for the ultra-compact 3D printed robot is solved to obtain the current control action by using current states and parameters of the system as initial state:

$$
\begin{array}{ll}
\min _{\xi(t), u(t)} & \frac{1}{2}\left\{\left\{\sum_{i=k+1}^{k+N_{c}-1}\left\|\xi_{r}\left(t_{i}\right)-\xi\left(t_{i}\right)\right\|_{Q_{k}}^{2}+\left\|u\left(t_{i}\right)\right\|_{R}^{2}\right\}\right. \\
& \left.+\left\|\xi_{r}\left(t_{k+N_{c}}\right)-\xi\left(t_{k+N_{c}}\right)\right\|_{Q_{N}}^{2}\right\} \\
\text { s.t. } \quad & \xi\left(t_{k}\right)=\hat{\xi}\left(t_{k}\right) \\
& p=\hat{p}\left(t_{\left.k-N_{e}+1\right)}\right. \\
& \dot{\xi}(t)=f(\xi(t), u(t), p) \\
& -0.1 \mathrm{rad} / s \leq \omega(t) \leq 0.1 \mathrm{rad} / s \quad t \in\left[t_{k}, t_{k+N-1}\right]
\end{array}
$$

where $Q_{k} \in \mathbb{R}^{n_{\xi} \times n_{\xi}}, R \in \mathbb{R}^{n_{u} \times n_{u}}$ and $Q_{N} \in \mathbb{R}^{n_{\xi} \times n_{\xi}}$ are symmetric and positive semi-definite weighting matrices, $\xi_{r}$ is the state reference, $\xi$ and $u$ are the states and inputs, $t_{k}$ is the current time, $N_{c}$ is the prediction horizon, $\hat{\xi}\left(t_{k}\right)$ is the current state estimates and $\hat{p}\left(t_{k-N_{e}+1}\right)$ is the current parameter estimates. The first term in the cost function is the stage cost and it is the cost throughout the prediction horizon. The second term in the cost function is the terminal penalty and it is the cost at the end of the prediction horizon. The terminal penalty is stated for stability reasons [23]. The weighting matrices $Q_{k}$, 
$R$ and $Q_{N}$ are selected as follows:

$$
Q_{k}=\operatorname{diag}(1,1,1), \quad R=1 \quad \text { and } \quad Q_{N}=10 \times Q_{k}
$$

The first element of the optimal control sequence is applied to the system:

$$
u\left(t_{k+1}, \xi_{k+1}\right)=u^{*}\left(t_{k+1}\right)
$$

and then the procedure is repeated for future sampling times by shifting prediction horizon for the subsequent time instant. It is important to remark that the control input $u^{*}\left(t_{k+1}\right)$ is precisely the same as it would be if all immeasurable states and parameters acquire values equal to their estimates based on the estimation up to current time $t_{k}$ due to the certainty equivalence principle.

The state reference for the 3D printed robot is dynamically changing online and defined as follows:

$$
\xi_{r}=\left[x_{r}, y_{r}, \theta_{r}\right]^{T}
$$

where $x_{r}$ and $y_{r}$ are the position references, $\omega_{r}$ is the yaw rate reference calculated from the position references as follows:

$$
\theta_{r}=\operatorname{atan} 2\left(\dot{y}_{r}, \dot{x}_{r}\right)+\lambda \pi
$$

where $\lambda$ describes the desired direction of the 3D printed field robot ( $\lambda=0$ for forward and $\lambda=1$ for backward).

\section{Solution Methods}

NMHE and NMPC methods for systems require online solutions of nonlinear least square optimization problems at each sampling time [24]. In this study, a single solution method, consisting of a fusion between multiple shooting and generalized Gauss-Newton methods, has been used to solve both optimization problems [25]. This approach is valid because the formulation of NMPC problem is akin to that NMHE problem. The generalized Gauss-Newton method derived from the classical Newton method was developed for least-squared problems. This method is advantageous because it does not require difficult computations of the second derivatives; however, it is challenging to foreknow the number of iterations to reach a solution of the desired accuracy. To overcome this challenge, the solution proposed in [26] has been used where the number of Gauss-Newton iterations is restricted to 1, and the initial value of each optimization problem takes on the value of the previous one intelligently. Hence, this improves the convergence of the Gauss-Newton method.

In this paper, the Gauss-Newton iteration is divided into two parts: preparation and feedback parts. The preparation parts are executed before the feedback parts, and the feedback part is executed after measurements for NMHE and estimates for NMPC are available. In the preparation part, the system dynamics are integrated with the previous solution, and objectives, constraints, and corresponding sensitives are evaluated. In the feedback part, a single quadratic program is solved with the current measurements for the NMHE and the current estimates for the NMPC. Thus, new estimates for the NMHE and new control signals for the NMPC are obtained. Compared to the classical method, this method minimizes feedback delay and produces similar results with higher computational efficiency.

\section{Corn Stand Counting Algorithm}

Conventional machine learning techniques require considerable domain-specific expertise to carefully design a feature extractor to transform raw data (e.g., pixel values of an image) into appropriate feature space where classifiers can detect patterns in the input. In contrast, Deep learning is a set of methods that allow end-to-end training and prediction. Deep learning models take raw data input and automatically learn the representations from the data's internal structure. Typically a deep learning model consists of multiple modules each of which slightly increases the level of abstraction from the previous representation. When enough such modules are used, very intricate structures can be learned such that relevant patterns are recognized whereas irrelevant variations are suppressed. Deep learning has made tremendous progress in areas that have confounded traditional machine learning for many years including image recognition [9], [11]. However, deep learning requires a lot of human-labeled images, unfortunately, this is something that is rather expensive to obtain. In this paper, we combine convolutional neural network (Section III-A) and support vector machine (Section III-B) via transfer learning to recognize corn plants using very few labeled images.

Recognition alone is not sufficient to count plants on a moving robot since it is difficult to distinguish different corn plants to avoid double-counting. To address this issue, we utilize motion estimation techniques to determine the number of plants that have passed through a fixed Region Of Interest (ROI). Details of this algorithm are given in Section III-D.

\section{A. Convolutional Neural Network}

It is important to maintain a good balance between performance and efficiency on algorithm to be implemented on mobile platforms, such as robots. MobileNets [27] is a family of networks specially developed for mobile and embedded applications. Due to the use of depthwise separable convolution that requires between 8 to 9 times less computation than standard convolution, the model runs significantly faster than its more complicated counterparts with only slight accuracy compromise. The network parameters are pre-trained on the ImageNet Large Scale Visual Recognition Competition (ILSVRC) dataset [28] with fully connected and softmax layer in the original model replaced by SVM for classification.

\section{B. Support Vector Machine}

Support Vector Machine (SVM) is a widely used model for classification [29]-[34]. The model constructs a hyperplane that maximizes the distance between the decision boundaries and any data point. The convexity of its loss function gives SVM desirable advantage over other methods such as feedforward neural network that suffers from the existence of multiple local minima and the need for a large dataset to ensure convergence [35], [36]. Therefore, we chose to replace the fully connected layer in the MobileNet with SVM 


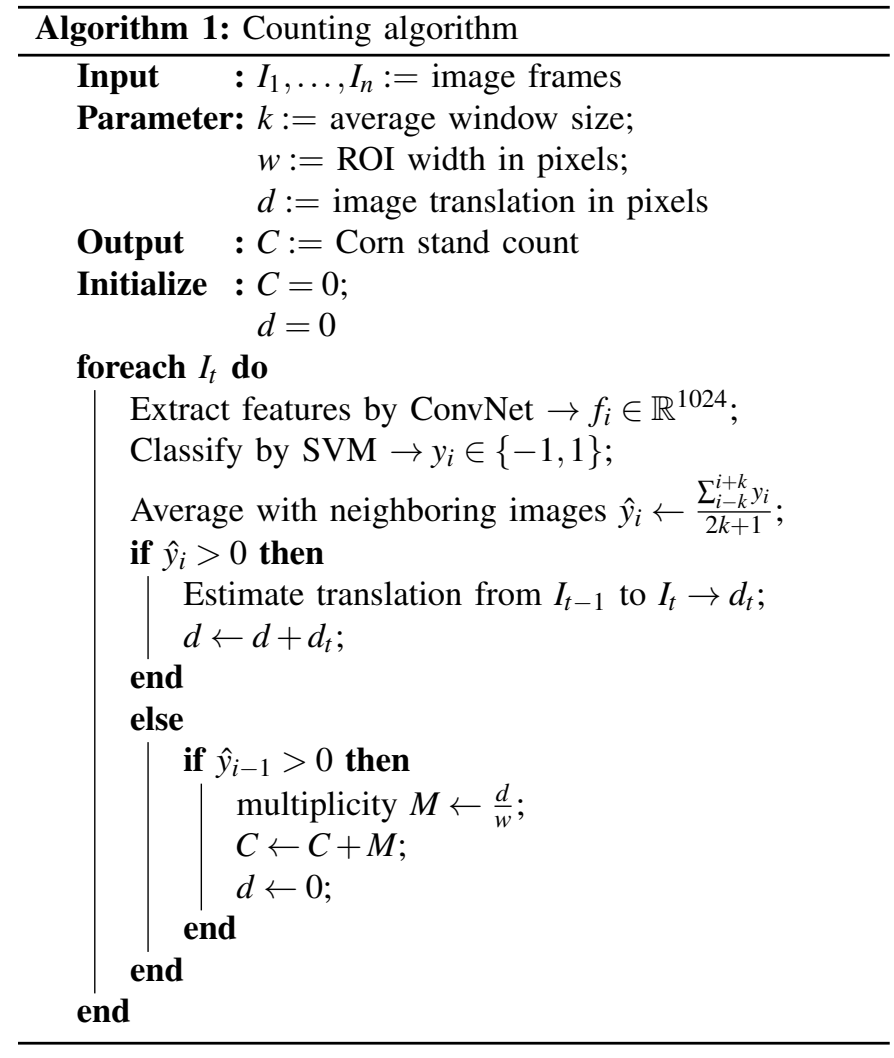

for better performance on limited data. The kernel functions of the SVM transform input data from the latent feature space of the deep network to a higher dimensional smooth Reproducing Kernel Hilbert Space. This enables the SVM to classify non-linearly separable features despite being a linearin-the-parameter classifier.

\section{Training}

Images are captured by the side facing camera on the robot throughout the growing season. The camera slightly points downward so that only the closest row is visible. Patches are cropped from the images and labeled according to the presence or absence of corn as positive and negative samples, respectively. The number of training and testing samples listed in Table I. We also employ data augmentation to increase the training data further. Images are rotated $\left( \pm 10^{\circ}\right)$, zoomed $(88 \%$ to $112 \%)$, vertically shifted $( \pm 15 \%)$, and horizontally flipped. Each image is augmented by 16-fold via randomly drawing transformations from the list.

The weights for the MobileNet are kept constant during transfer learning. The hyper-parameter for SVM is determined by grid search and cross-validation implemented in Python package scikit-learn [37]. Linear kernel and Radial Basis Function (RBF) kernel are investigated. The values of hyperparameters that we consider in this study are listed Table II . All combinations are exhausted to identify the optimal parameters.
TABLE I

NUMBER OF TRAINING AND TESTING SAMPLE AT EACH GROWTH STAGE.

\begin{tabular}{l|l|l|l|l|l} 
& & $\mathrm{V} 4$ & $\mathrm{VT}$ & $\mathrm{R} 2$ & $\mathrm{R} 6$ \\
\hline \multirow{2}{*}{ Training } & positive & 348 & 328 & 519 & 263 \\
\cline { 2 - 6 } & negative & 416 & 304 & 310 & 291 \\
\hline \multirow{2}{*}{ Testing } & positive & 89 & 83 & 130 & 66 \\
\cline { 2 - 6 } & negative & 105 & 77 & 78 & 73
\end{tabular}

TABLE II

SVM HYPER-PARAMETERS TESTED IN THE GRID SEARCH.

\begin{tabular}{c|c|c} 
& Linear & RBF \\
\hline$C$ & $1,10,100,1000$ & $1,10,100,1000$ \\
\hline$\gamma$ & N/A & $10^{-2}, 10^{-3}, 10^{-4}, 10^{-5}$
\end{tabular}

\section{Motion Estimate}

Instead of classifying the whole image, we focus on a fixed region of interest (ROI) whose size is slightly less than the average plant spacing. In the majority of the time, only single corn plant appears in the ROI as in Figure 2(a). The classification results then form a time series of alternating step function where each plateau represents a corn plant. However, when the gap between two neighboring plants is smaller than the ROI, we compute the horizontal translation $T$ between two consecutive frames $I_{1}$ and $I_{2}$ when a positive signal of the binary classification is present. If the ROI finishes scanning through a corn before encountering the next one as in most cases, $T$ should approximately equal to ROI width $w$. In the case that adjacent corn plants are close, $T=(n-1) d+w$, where $n$ is the number of plants, $d$ is the distance between neighboring plants as shown in Fig. 2(b). If we further assume that $d$ is not much smaller than $w$, then $n \approx \frac{T}{w}$. In real-time implementations, taking the ratio results in a floating point number that is rounded towards the nearest integer. In other words, the inequality (11) must be held. For instance $n=2$, the algorithm can correctly count two nearby corn plants as long as their distance is not smaller than half of the ROI width. The lower bound on $d$ increases as the number of adjacent corn plants $n$. But based on our observation, in most cases $n$ is less than 5.

$$
\begin{gathered}
n-0.5 \leq \frac{T}{w} \leq n+0.5, \quad \frac{n-1.5}{n-1} \leq \frac{d}{w} \leq \frac{n-0.5}{n-1} \\
\text { IV. FIELD RESUlts }
\end{gathered}
$$

\section{A. Real-time Results for Navigation}

Linear controllers cannot achieve good trajectory tracking performance on uneven terrain if the system starts off-track [38]. As shown in Fig. 3(a), after the 3D printed field robot is started off-track, it is capable of reaching to the reference trajectory and staying on-track. One of the major problems of such GNSS-based navigated outdoor robots is the missing data values from the satellites. This drawback is inevitable for RealTime Kinematic (RTK) GNSSs, and the control algorithm has 


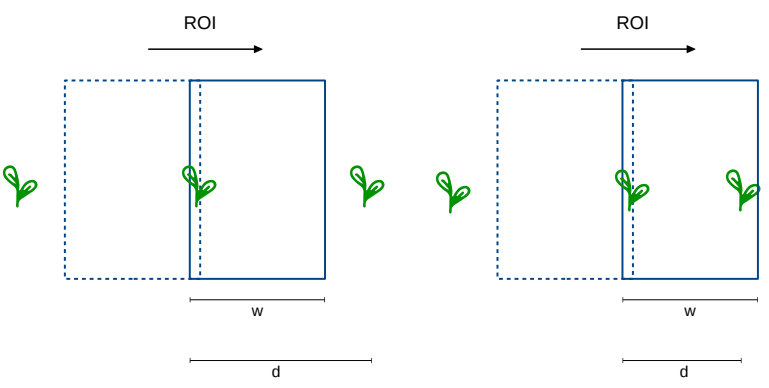

(a) Single corn appears in the ROI (b) Two corn plants appear in the at a time $(w<d)$

ROI at the same time $(w \geq d)$

Fig. 2. Examples of: (a) normal cases where a single corn appears in the ROI. The relative motion between the camera and corn $T \approx w$; (b) exceptions where neighboring corn plants are too close to be separated In this case, $T \approx d+w$.

to be robust to cope with missing data points. As can be observed from Fig. 3(a), although there were 35 missing data points within 1850 data points throughout the experiment, high accurate trajectory tracking performance has been achieved by the NMHE-NMPC framework.

The Euclidean error is shown in Fig. 3(b). The mean value of the Euclidean error after staying on-track is $0.0423 \mathrm{~m}$. The NMPC benefits from traction parameters estimated by the NMHE and results in highly accurate tracking performance so that the Euclidean error is less than $10 \mathrm{~cm}$. The number of violations is shown in Fig. 3(c). The results of multiple experiments indicate that the NMHE-NMPC framework does not violate this error constraint. This demonstrates the capability of the NMHE-NMPC framework.

The traction parameters estimates are shown in 3(d) and within the upper and lower bounds specified in (6). It is clearly seen that they consistently stay close the upper bound and stabilize at values which ensure stable trajectory. It is to be noted that if the soil conditions are unchanging, then hard-coding might result in more accurate trajectory tracking performance. However, the soil conditions are dynamically changing in practice.

The measured and estimated speeds of the ultra-compact 3D printed field robot are shown in Fig. 3(e). As can be seen, the robot has a constant speed, and the NMHE is capable of dealing with noise on the measurements. It is to be noted that some estimated speed values between 300 and 350 seconds are larger than measurements. The reason is that there are missing GNSS measurements as shown in Fig. 3(a). If GNSS measurement is invalid, previous valid measurement is fed to the NMHE instead of an outlier. There are 14 invalid points in the GNSS measurements between 318.8 and 321.6 seconds and these result in the peak values in the speed estimates between 322.8 and 325.6 seconds. The effect on the speed estimates arises seen subsequently because the speed in the system model (2) is considered as a parameter and the parameter estimation is achieved in the arrival cost of the NMHE while the parameters in the estimation horizon are assumed to be time-invariant. Moreover, the longitudinal traction parameter estimate in Fig. 3(d) has nadir values between 322.8 and 325.6 seconds similar to the speed estimate. Consequently, the effective speed $\mu v$ is equal to the speed of the robot despite unavailable GNSS points. The same explanation is the case for the two peak points the speed estimate between after around 340 seconds.

The output of the NMPC, i.e., the desired yaw rate, and yaw rate measurements are shown in Fig. 3(f). The generated control signal by the NMPC is within the lower and upper bounds specified in (6). The performance of the low-level controller is sufficient to track the reference signals despite the high noisy measurements.

\section{B. Real-Time Results for Corn Stand Counting}

We collected data from various corn fields. The dates and growth stages when data were collected span the entire growing season. In each field, we randomly selected a row and drove the robot at a constant velocity for an arbitrary number of plots. The true populations were counted manually. For data collections prior to October, a small portion of the images were annotated. We collected two additional data-sets in October to test the generalization ability of the model on unseen data from different fields conditions. Table III summarizes the conditions of the data collection. We follow the widely used staging method by Abendroth et al. [39]. The method divides the growth of corn into vegetative phase and reproductive phase. The vegetative phase covers stages from emergence (VE) to tasseling (VT), where stages in-between are identified by the number of collar leaves (e.g. V4). The reproductive phase includes silking (R1), blister (R2), milk (R3), dough (R4), dent (R5), and maturity (R6).

1) SVM training: The best hyper-parameters for the SVM found in the grid search are listed in Table IV with their performance metrics on the test data. The hyper-parameters are almost the same for the four growth stages except that the $\mathrm{C}$-value is different for V4, largely due to the difference in appearance of corn plants at V4 from VT, R2, and R6. The RBF kernel consistently works better than the linear kernel, and the optimal $\gamma$-value for the kernel is identical at four stages.

The performance metrics are also consistently high for different growth stages. It is noted the training samples exclude leaves and only focus on stems and stalks. The metrics demonstrate that the deep learning model is capable of distinguishing subtle difference in images and thus reduces the inference of leaves and weeds. The mean accuracy for all stages is $91.67 \%$.

2) Validation: In-Field Corn counting: Figure 4 shows the corn plant population per plot by robot vs human for each dataset. The robot predictions agree well with the ground truth. The least-square fitted line through all data is given as count $_{\text {robot }}=0.96 \times$ count $_{\text {human }}+0.85$ and a correlation coefficient $R=0.96$.

Figure 5 shows the box-and-whisker plot of the relative accuracy as in Equation (12) for each dataset. The algorithm achieves consistently high accuracy for all locations and growth stages. It is noted that data from Martinsville and Lebanon were processed without using the new data 


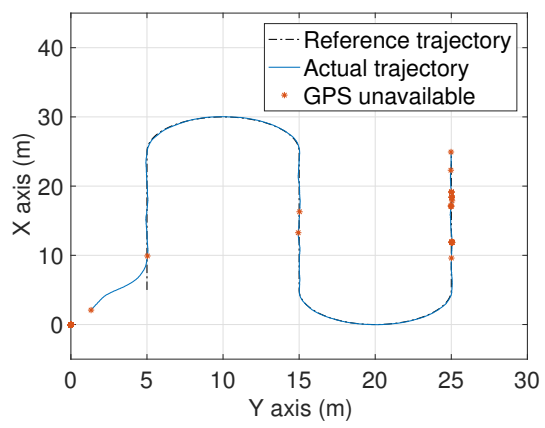

(a)
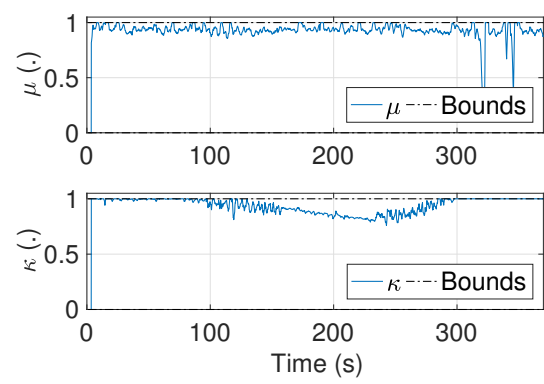

(d)

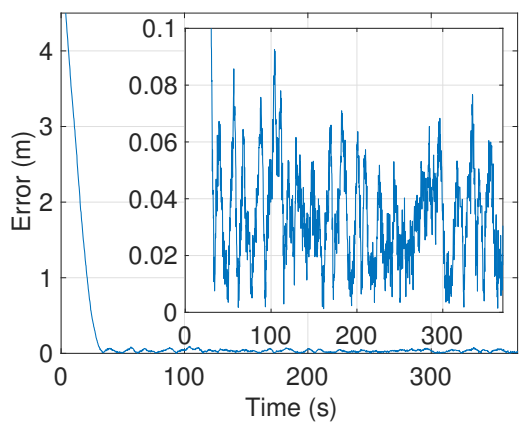

(b)

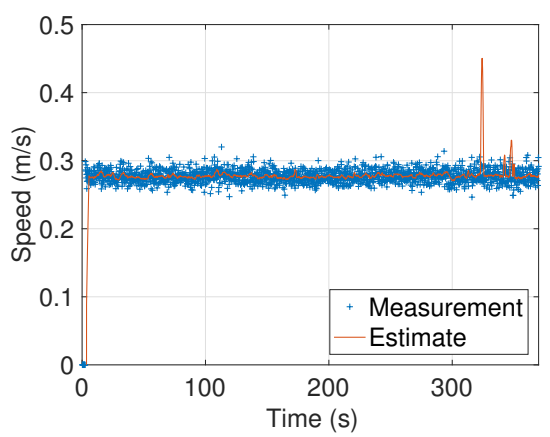

(e)

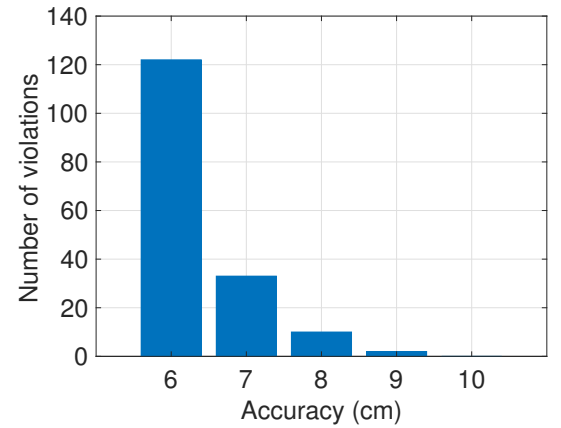

(c)

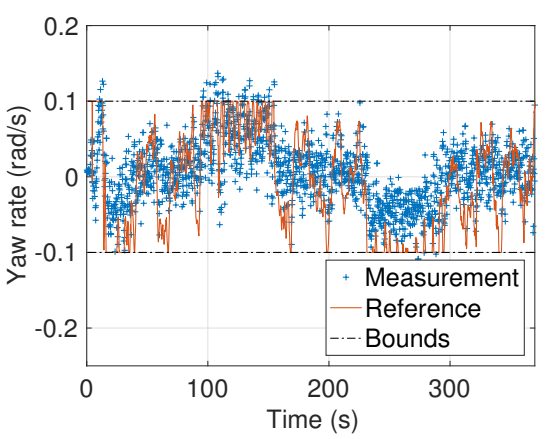

(f)

Fig. 3. a) Reference and actual trajectories, and unavailable Global Navigation Satellite System (GNSS) signals on the trajectory. b) Euclidean error. The mean value of Euclidean error is less than $5 \mathrm{~cm}$ after being stayed on-track. c) The number of violations. The available space on either side of the field robot is restricted to $0.12 \mathrm{~m}$. It does not violate this accuracy limit. d) Reference and estimated yaw angles, and estimated traction parameters $\mu$, $\kappa$. e) Measured and estimated speeds. f) Reference and measured yaw rates.

TABLE III

FIELD CONDITIONS FOR EACH DATA COLLECTION, INCLUDING DATE AND LOCATION OF THE FIELD, WHETHER DATA ARE SAMPLED FOR TRAINING IMAGE RECOGNITION MODEL, THE GROWTH STAGE OF CORN PLANTS AT COLLECTION TIME, AND A NUMBER OF PLOTS COLLECTED.

\begin{tabular}{|c|c|c|c|c|}
\hline Date & Location & Training & Growth stage & Plots \\
\hline Jun $6^{\text {th }}, 2017$ & Assumption, IL & Yes & V4 & 16 \\
\hline Jul $6^{\text {th }}, 2017$ & Champaign, IL & Yes & VT & 10 \\
\hline Aug $2^{\text {nd }}, 2017$ & Champaign, IL & Yes & $\mathrm{R} 2$ & 28 \\
\hline Sep $21^{\text {st }}, 2017$ & Ivesdale, IL & Yes & R6 & 10 \\
\hline Oct $25^{\text {th }}, 2017$ & Lebanon, ID & No & V6 & 15 \\
\hline Oct $26^{\text {th }}, 2017$ & Martinsville, IL & No & R6 & 8 \\
\hline
\end{tabular}

TABLE IV

BEST HYPER-PARAMETERS FOUND IN THE GRID SEARCH AND THEIR CORRESPONDING PERFORMANCE METRICS ON THE TEST DATA.

\begin{tabular}{lcccc}
\hline & V4 & VT & R2 & R6 \\
\hline kernel & rbf & rbf & rbf & rbf \\
\hline $\mathrm{C}$ & 10 & 100 & 100 & 100 \\
\hline$\gamma$ & 0.001 & 0.001 & 0.001 & 0.001 \\
\hline accuracy & $91.75 \%$ & $94.38 \%$ & $92.79 \%$ & $87.77 \%$ \\
\hline precision & 0.91 & 0.91 & 0.91 & 0.89 \\
\hline recall & 0.95 & 0.94 & 0.95 & 0.88 \\
\hline F1-score & 0.95 & 0.93 & 0.94 & 0.88 \\
\hline
\end{tabular}

to train SVM. The results demonstrate that the recognition model generalizes well to unseen data and handles real-world variations effectively.

$$
\text { accuracy }=1-\frac{\mid \text { count }_{\text {robot }}-\text { count }_{\text {human }} \mid}{\text { count }_{\text {human }}}
$$

\section{CONCLUSiOns}

The embedded high precision control and corn stand counting algorithms for a low-cost, ultra-compact 3D printed, autonomous field robot for off-road terrain have been elaborated in this paper.

An NMHE that identifies key terrain parameters using onboard robot sensors and a learning-based NMPC that enables highly accurate tracking have been developed to be capable of autonomously and safely navigating through row based crops. The developed framework for high precision control that enables reliable field robot path tracking in off-road terrain provides less than $5 \mathrm{~cm}$ accuracy in the presence of slip and does not violate the $12 \mathrm{~cm}$ accuracy limit to avoid crop damage.

The developed vision algorithm based on color imagery recognizes corn stalks robustly in the presence of interference from leaves and weeds, yet only requires relatively small amount of data for training to estimate corn plant population. This was achieved by fusing a leading deep learning model 


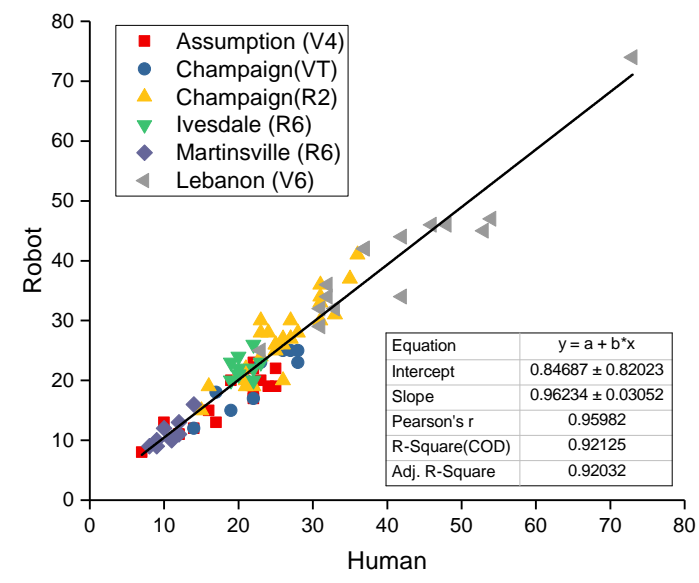

Fig. 4. Scatter plot of corn plants per plot counted by robot vs human for each dataset. The line represents a linear fit through all data. The robot predictions agree well with the ground truth with the correlation coefficient $R=0.96$.

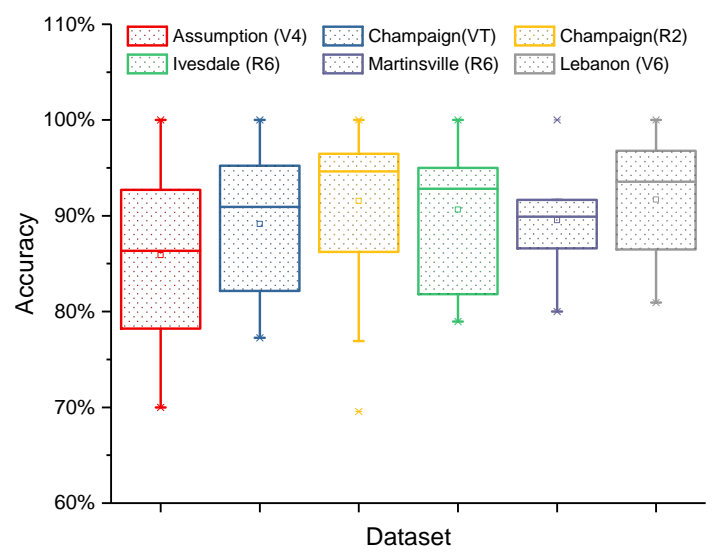

Fig. 5. Box plot of the accuracy as in Equation (12) for each dataset. The accuracy stays consistent for different locations and growth stages even for datasets from Martinsville and Lebanon where no new training data are used.

with Support Vector Machine Classifiers. Our method was tested at five different growth stages and locations. The robot predictions agreed well with the ground truth with count $_{\text {robot }}=$ $0.96 \times$ count $_{\text {human }}+0.85$. Least-square regression shows strong correlation $(R=0.96)$ between populations given by robot and human. In addition, the accuracy stays consistently high for all growth stages and locations. These results establish that ultra-compact, lightweight, autonomous agricultural robots equipped with exteroceptive sensors and artificial intelligence could be utilized to relieve the phenotyping bottleneck.

\section{ACKNOWLEDGMENTS}

The information, data, or work presented herein was funded in part by the Advanced Research Projects Agency-Energy
(ARPA-E), U.S. Department of Energy, under Award Number DE-AR0000598. The views and opinions of authors expressed herein do not necessarily state or reflect those of the United States Government or any agency thereof.

Dr. Erkan Kayacan is a postdoctoral researcher at Massachusetts Institute of Technology since February 2018. Any questions or comments should be directed to Girish Chowdhary. We thank EarthSense Inc. for valuable suggestions.

The authors would like to thank Benjamin Thompson and others at Distributed Autonomous Systems Laboratory for valuable contributions to the design and construction of the TerraSentia robot.

\section{REFERENCES}

[1] R. T. Furbank and M. Tester, "Phenomics-technologies to relieve the phenotyping bottleneck," Trends in plant science, vol. 16, no. 12, pp. 635-644, 2011.

[2] J. L. Araus and J. E. Cairns, "Field high-throughput phenotyping: the new crop breeding frontier," Trends in plant science, vol. 19, no. 1, pp. 52-61, 2014.

[3] E. J. van Henten, D. Goense, and C. Lokhorst, Precision agriculture '09, D. Goense, E. J. van Henten, and C. Lokhorst, Eds. The Netherlands: Wageningen Academic Publishers, 2009.

[4] P. Biber, U. Weiss, M. Dorna, and A. Albert, "Navigation system of the autonomous agricultural robot bonirob," in Workshop on Agricultural Robotics: Enabling Safe, Efficient, and Affordable Robots for Food Production (Collocated with IROS 2012), Vilamoura, Portugal, 2012.

[5] A. Shafiekhani, S. Kadam, F. B. Fritschi, and G. N. DeSouza, "Vinobot and vinoculer: Two robotic platforms for high-throughput field phenotyping," Sensors, vol. 17, no. 1, 2017.

[6] T. Mueller-Sim, M. Jenkins, J. Abel, and G. Kantor, "The robotanist: A ground-based agricultural robot for high-throughput crop phenotyping," in 2017 IEEE International Conference on Robotics and Automation (ICRA), May 2017, pp. 3634-3639.

[7] E. Kayacan, E. Kayacan, H. Ramon, and W. Saeys, "Distributed nonlinear model predictive control of an autonomous tractortrailer system," Mechatronics, vol. 24, no. 8, pp. 926 - 933, 2014.

[8] K. He, X. Zhang, S. Ren, and J. Sun, "Deep residual learning for image recognition," in Proceedings of the IEEE conference on computer vision and pattern recognition, 2016, pp. 770-778.

[9] A. Krizhevsky, I. Sutskever, and G. E. Hinton, "Imagenet classification with deep convolutional neural networks," in Advances in neural information processing systems, 2012, pp. 1097-1105.

[10] C. Szegedy, W. Liu, Y. Jia, P. Sermanet, S. Reed, D. Anguelov, D. Erhan, V. Vanhoucke, and A. Rabinovich, "Going deeper with convolutions," in Proceedings of the IEEE conference on computer vision and pattern recognition, 2015, pp. 1-9.

[11] K. Simonyan and A. Zisserman, "Very deep convolutional networks for large-scale image recognition," arXiv preprint arXiv:1409.1556, 2014.

[12] K. Iagnemma, S. Kang, H. Shibly, and S. Dubowsky, "Online terrain parameter estimation for wheeled mobile robots with application to planetary rovers," IEEE Transactions on Robotics, vol. 20, no. 5, pp. 921-927, Oct 2004

[13] L. E. Ray, "Estimation of terrain forces and parameters for rigid-wheeled vehicles," IEEE Transactions on Robotics, vol. 25, no. 3, pp. 717-726, June 2009.

[14] E. Kayacan, E. Kayacan, H. Ramon, and W. Saeys, "Modeling and identification of the yaw dynamics of an autonomous tractor," in 2013 9th Asian Control Conference (ASCC), June 2013, pp. 1-6.

[15] S. U. Lee and K. Iagnemma, "Robust motion planning methodology for autonomous tracked vehicles in rough environment using online slip estimation," in 2016 IEEE/RSJ International Conference on Intelligent Robots and Systems (IROS), Oct 2016, pp. 3589-3594.

[16] E. Kayacan, E. Kayacan, H. Ramon, and W. Saeys, "Nonlinear modeling and identification of an autonomous tractor-trailer system," Computers and Electronics in Agriculture, vol. 106, pp. 1 - 10, 2014.

[17] S. U. Lee, R. Gonzalez, and K. Iagnemma, "Robust sampling-based motion planning for autonomous tracked vehicles in deformable high slip terrain," in 2016 IEEE International Conference on Robotics and Automation (ICRA), May 2016, pp. 2569-2574. 
[18] E. Kayacan, E. Kayacan, H. Ramon, and W. Saeys, "Robust tubebased decentralized nonlinear model predictive control of an autonomous tractor-trailer system," IEEE/ASME Transactions on Mechatronics, vol. 20, no. 1, pp. 447 - 456, Feb 2015.

[19] E. L. Haseltine and J. B. Rawlings, "Critical evaluation of extended kalman filtering and moving-horizon estimation," Industrial \& Engineering Chemistry Research, vol. 44, no. 8, pp. 2451-2460, 2005.

[20] H. Ferreau, T. Kraus, M. Vukov, W. Saeys, and M. Diehl, "High-speed moving horizon estimation based on automatic code generation," in Decision and Control (CDC), 2012 IEEE 51st Annual Conference on, 2012, pp. 687-692.

[21] E. Kayacan, E. Kayacan, H. Ramon, and W. Saeys, "Towards agrobots: Identification of the yaw dynamics and trajectory tracking of an autonomous tractor," Computers and Electronics in Agriculture, vol. 115, pp. $78-87,2015$.

[22] E. Kayacan, E. Kayacan, I.-M. Chen, H. Ramon, and W. Saeys, On the Comparison of Model-Based and Model-Free Controllers in Guidance, Navigation and Control of Agricultural Vehicles. Cham: Springer International Publishing, 2018, pp. 49-73.

[23] D. Mayne, J. Rawlings, C. Rao, and P. Scokaert, "Constrained model predictive control: Stability and optimality," Automatica, vol. 36, no. 6, pp. $789-814,2000$.

[24] E. Kayacan, J. M. Peschel, and E. Kayacan, "Centralized, decentralized and distributed nonlinear model predictive control of a tractor-trailer system: A comparative study," in 2016 American Control Conference (ACC), July 2016, pp. 4403-4408.

[25] E. Kayacan, E. Kayacan, H. Ramon, and W. Saeys, "Learning in centralized nonlinear model predictive control: Application to an autonomous tractor-trailer system," IEEE Transactions on Control Systems Technology, vol. 23, no. 1, pp. 197-205, Jan 2015.

[26] M. Diehl, H. Bock, J. P. Schlder, R. Findeisen, Z. Nagy, and F. Allgöwer, "Real-time optimization and nonlinear model predictive control of processes governed by differential-algebraic equations," Journal of Process Control, vol. 12, no. 4, pp. 577 - 585, 2002.

[27] A. G. Howard, M. Zhu, B. Chen, D. Kalenichenko, W. Wang, T. Weyand, M. Andreetto, and H. Adam, "Mobilenets: Efficient convolutional neural networks for mobile vision applications," arXiv preprint arXiv:1704.04861, 2017.

[28] J. Pruegsanusak and A. Howard, https://github.com/tensorflow/models/ blob/master/slim/nets/mobilenet_v1.md, accessed: 2017-08-12.

[29] N. Cristianini and J. Shawe-Taylor, "An introduction to support vector machines," 2000.

[30] B. Scholkopf, K.-K. Sung, C. J. Burges, F. Girosi, P. Niyogi, T. Poggio, and V. Vapnik, "Comparing support vector machines with gaussian kernels to radial basis function classifiers," IEEE transactions on Signal Processing, vol. 45, no. 11, pp. 2758-2765, 1997.

[31] A. J. Smola and B. Schölkopf, "On a kernel-based method for pattern recognition, regression, approximation, and operator inversion," Algorithmica, vol. 22, no. 1-2, pp. 211-231, 1998.

[32] A. J. Smola, B. Schölkopf, and K.-R. Müller, "The connection between regularization operators and support vector kernels," Neural networks, vol. 11, no. 4, pp. 637-649, 1998.

[33] B. Scholkopf, S. Mika, C. J. Burges, P. Knirsch, K.-R. Muller, G. Ratsch and A. J. Smola, "Input space versus feature space in kernel-based methods," IEEE transactions on neural networks, vol. 10, no. 5, pp 1000-1017, 1999.

[34] B. Schölkopf, C. J. Burges, and A. J. Smola, Advances in kernel methods: support vector learning. MIT press, 1999.

[35] J. A. Suykens, J. De Brabanter, L. Lukas, and J. Vandewalle, "Weighted least squares support vector machines: robustness and sparse approximation," Neurocomputing, vol. 48, no. 1, pp. 85-105, 2002.

[36] C. M. Bishop, Neural networks for pattern recognition. Oxford university press, 1995.

[37] F. Pedregosa, G. Varoquaux, A. Gramfort, V. Michel, B. Thirion, O. Grisel, M. Blondel, P. Prettenhofer, R. Weiss, V. Dubourg, J. Vanderplas, A. Passos, D. Cournapeau, M. Brucher, M. Perrot, and E. Duchesnay, "Scikit-learn: Machine learning in Python," Journal of Machine Learning Research, vol. 12, pp. 2825-2830, 2011.

[38] E. Kayacan, H. Ramon, and W. Saeys, "Robust trajectory tracking error model-based predictive control for unmanned ground vehicles," IEEE/ASME Transactions on Mechatronics, vol. 21, no. 2, pp. 806-814, April 2016.

[39] L. J. Abendroth, R. W. Elmore, M. J. Boyer, and S. K. Marlay, "Corn growth and development," 2011. 